\section{Improved Delay-Dependent Stability Criteria for Time-Delay Systems}

Shengyuan Xu and James Lam

\begin{abstract}
This note provides an improved asymptotic stability condition for time-delay systems in terms of a strict linear matrix inequality. Unlike previous methods, the mathematical development avoids bounding certain cross terms which often leads to conservatism. When time-varying norm-bounded uncertainties appear in a delay system, an improved robust delay-dependent stability condition is also given. Examples are provided to demonstrate the reduced conservatism of the proposed conditions.
\end{abstract}

Index Terms-Delay-dependent condition, linear matrix inequality (LMI), time-delay systems, uncertain systems.

\section{INTRODUCTION}

Over the past decades, there has been a growing interest in the stability analysis of time-delay systems since time delays are often the sources of instability and encountered in various engineering systems such as chemical processes, long transmission lines in pneumatic systems [3]. Existing criteria for asymptotic stability of time-delay systems can be classified into two types; that is, delay-independent stability and delay-dependent stability [3], [12]; the former does not include any information on the size of delay while the latter employs such information. It is known that delay-dependent stability conditions are generally less conservative than delay-independent ones especially when the size of the delay is small. A great number of delay-dependent stability results have been reported in the literature; see, e.g. [1], [9]-[11], and the references therein. All these stability results provide sufficient conditions and have, to some yet different extent, conservatism. It is now known that the conservatism of the delay-dependent conditions stems from two causes; the first is the way adopted to transform the original delay model to an appropriate one for analysis and the second is the method for evaluating the bounds on some weighted cross products arising in the analysis of the delay-dependent stability problem. Over the past decade, a large number of results have been published aiming at reducing the conservatism of stability criteria. It is generally recognized that to further improve the quality of delay-dependent stability criteria is a very difficult task. The aim of this note is to provide a further contribution to improving delay-dependent stability conditions.

In this note, an enhanced delay-dependent stability condition for linear systems with time-invariant delays is obtained in terms of a strict linear matrix inequality (LMI). Compared to earlier works, the theoretical development is simpler and, unlike previous methods in deriving the delay-dependent stability criteria in the literature, an upper bounding on the weighted cross products of the state and the delayed state is avoided (and hence no inequalities are needed for bounding these cross terms). It is worth pointing out that increasing attention has been focused on the improvement of such bounding technique in the

Manuscript received July 23, 2003; revised January 28, 2004, June 6, 2004, and November 20, 2004. Recommended by Associate Editor Y. Ohta. This work was supported by CRCG 10205878, the Foundation for the Author of National Excellent Doctoral Dissertation of P. R. China under Grant 200240, the National Natural Science Foundation of P. R. China under Grant 60304001, and the Fok Ying Tung Education Foundation under Grant 91061.

$\mathrm{S}$. Xu is with the Department of Automation, Nanjing University of Science and Technology, Nanjing 210094, P. R. China.

J. Lam is with the Department of Mechanical Engineering, University of Hong Kong, Hong Kong.

Digital Object Identifier 10.1109/TAC.2005.843873 past which, by the present work, is shown to be unnecessary. Also, no model transformation is involved in the derivation of our stability condition. It is theoretically established that the stability result in this note is less conservative than that in [9]. Based on the derived delay-dependent stability condition, an improved delay-dependent robust stability condition is proposed for delay systems with time-varying normbounded parameter uncertainties. Examples are provided to demonstrate the less conservatism of the obtained results.

Notation: Throughout this note, for real symmetric matrices $X$ and $Y$, the notation $X \geq Y$ (respectively, $X>Y$ ) means that the matrix $X-Y$ is positive semidefinite (respectively, positive definite). $I$ is an identity matrix with appropriate dimension. The superscript " $T$ " represents the transpose. The notation $|\cdot|$ refers to the Euclidean vector norm. We use $\lambda_{\min }(\cdot)$ to denote the minimum eigenvalue of a symmetric matrix. Matrices, if not explicitly stated, are assumed to have compatible dimensions.

\section{Main Results}

Consider the following time-delay system:

$$
\begin{aligned}
(\Sigma): \quad \dot{x}(t) & =A x(t)+A_{h} x(t-h) \\
x(t) & =\phi(t) \quad \forall t \in[-h, 0]
\end{aligned}
$$

where $x(t) \in \mathbb{R}^{n}$ is the state, and $\phi(t)$ is the initial condition. The scalar $h>0$ is the delay of the system, $A$ and $A_{h}$ are known real constant matrices.

The following theorem gives an improved delay-dependent stability for system $(\Sigma)$.

Theorem 1: The time-delay system $(\Sigma)$ is asymptotically stable for any delay $h$ satisfying $0<h \leq \bar{h}$ if there exist matrices $P>0$, $Q>0, Z>0, Y$ and $W$ such that the LMI shown in (3) at the bottom of the next page holds.

Proof: Denote

$$
x_{t}=x(t+\theta) \quad-2 h \leq \theta \leq 0
$$

and define a Lyapunov functional candidate for the time-delay system $(\Sigma)$ as

$$
V\left(x_{t}\right)=V_{1}\left(x_{t}\right)+V_{2}\left(x_{t}\right)+V_{3}\left(x_{t}\right)
$$

where

$$
\begin{aligned}
& V_{1}\left(x_{t}\right)=x(t)^{T} \operatorname{Px}(t) \\
& V_{2}\left(x_{t}\right)=\int_{-h}^{0} \int_{t+\beta}^{t} \dot{x}(\alpha)^{T} Z \dot{x}(\alpha) d \alpha d \beta \\
& V_{3}\left(x_{t}\right)=\int_{t-h}^{t} x(\alpha)^{T} Q x(\alpha) d \alpha .
\end{aligned}
$$

Then, by the Newton-Leibniz formula, we have

$$
x(t-h)=x(t)-\int_{t-h}^{t} \dot{x}(\alpha) d \alpha
$$

and

$$
\begin{aligned}
\dot{V}_{1}\left(x_{t}\right) & =2 x(t)^{T} P\left[A x(t)+A_{h} x(t-h)\right] \\
& =2 x(t)^{T} P\left(A+A_{h}\right) x(t)-2 x(t)^{T} P A_{h} \int_{t-h}^{t} \dot{x}(\alpha) d \alpha
\end{aligned}
$$




$$
\begin{aligned}
& =2 x(t)^{T} P\left(A+A_{h}\right) x(t)+2 x(t)^{T}\left(Y-P A_{h}\right) \\
& \times \int_{t-h}^{t} \dot{x}(\alpha) d \alpha+2 x(t-h)^{T} W \int_{t-h}^{t} \dot{x}(\alpha) d \alpha \\
& -\left[2 x(t)^{T} Y \int_{t-h}^{t} \dot{x}(\alpha) d \alpha\right. \\
& \left.+2 x(t-h)^{T} W \int_{t-h}^{t} \dot{x}(\alpha) d \alpha\right] \\
& =\frac{1}{h} \int_{t-h}^{t}\left[2 x(t)^{T}(P A+Y) x(t)\right. \\
& +2 x(t)^{T}\left(P A_{h}-Y+W^{T}\right) x(t-h) \\
& -2 x(t-h)^{T} W x(t-h)-2 x(t)^{T} h Y \dot{x}(\alpha) \\
& \left.-2 x(t-h)^{T} h W \dot{x}(\alpha)\right] d \alpha \\
& \dot{V}_{2}\left(x_{t}\right)=\int_{-h}^{0}\left[\dot{x}(t)^{T} Z \dot{x}(t)-\dot{x}(t+\beta)^{T} Z \dot{x}(t+\beta)\right] d \beta \\
& =\int_{t-h}^{t}\left[\dot{x}(t)^{T} Z \dot{x}(t)-\dot{x}(\alpha)^{T} Z \dot{x}(\alpha)\right] d \alpha \\
& =\int_{t-h}^{t}\left[\left(A x(t)+A_{h} x(t-h)\right)^{T} Z\right. \\
& \left.\times\left(A x(t)+A_{h} x(t-h)\right)-\dot{x}(\alpha)^{T} Z \dot{x}(\alpha)\right] d \alpha \\
& =\frac{1}{h} \int_{t-h}^{t}\left[x(t)^{T} h A^{T} Z A x(t)\right. \\
& +2 x(t)^{T} h A^{T} Z A_{h} x(t-h) \\
& +x(t-h)^{T} h A_{h}^{T} Z A_{h} x(t-h) \\
& \left.-\dot{x}(\alpha)^{T} h Z \dot{x}(\alpha)\right] d \alpha
\end{aligned}
$$

and

$$
\begin{aligned}
\dot{V}_{3}\left(x_{t}\right) & =x(t)^{T} Q x(t)-x(t-h)^{T} Q x(t-h) \\
& =\frac{1}{h} \int_{t-h}^{t}\left[x(t)^{T} Q x(t)-x(t-h)^{T} Q x(t-h)\right] d \alpha .
\end{aligned}
$$

It then follows from (4)-(6) that

$$
\dot{V}\left(x_{t}\right)=\frac{1}{h} \int_{t-h}^{t} \zeta(t, \alpha)^{T} \Lambda(h) \zeta(t, \alpha) d \alpha
$$

where $\zeta(t, \alpha)$ and $\Lambda(h)$ are defined at the bottom of the page holds true. Now, applying the Schur complement equivalence to (3) gives that $Z>0$ and

$$
\begin{aligned}
& {\left[\begin{array}{rr}
P A+A^{T} P+Y+Y^{T}+Q & P A_{h}-Y+W^{T} \\
A_{h}^{T} P-Y^{T}+W & -Q-W-W^{T}
\end{array}\right]+} \\
& \bar{h}\left[\begin{array}{cc}
-Y & A^{T} Z \\
-W & A_{h}^{T} Z
\end{array}\right]\left[\begin{array}{cc}
Z^{-1} & 0 \\
0 & Z^{-1}
\end{array}\right]\left[\begin{array}{cc}
-Y^{T} & -W^{T} \\
Z A & Z A_{h}
\end{array}\right]<0 .
\end{aligned}
$$

Therefore, for all $h$ satisfying $0<h \leq \bar{h}$, we have

$$
\begin{aligned}
& {\left[\begin{array}{cc}
P A+A^{T} P+Y+Y^{T}+Q & P A_{h}-Y+W^{T} \\
A_{h}^{T} P-Y^{T}+W & -Q-W-W^{T}
\end{array}\right]} \\
& +h\left[\begin{array}{cc}
-Y & A^{T} Z \\
-W & A_{h}^{T} Z
\end{array}\right]\left[\begin{array}{cc}
Z^{-1} & 0 \\
0 & Z^{-1}
\end{array}\right]\left[\begin{array}{cc}
-Y^{T} & -W^{T} \\
Z A & Z A_{h}
\end{array}\right] \\
& \leq\left[\begin{array}{cc}
P A+A^{T} P+Y+Y^{T}+Q & P A_{h}-Y+W^{T} \\
A_{h}^{T} P-Y^{T}+W & -Q-W-W^{T}
\end{array}\right] \\
& +\bar{h}\left[\begin{array}{cc}
-Y & A^{T} Z \\
-W & A_{h}^{T} Z
\end{array}\right]\left[\begin{array}{cc}
Z^{-1} & 0 \\
0 & Z^{-1}
\end{array}\right]\left[\begin{array}{cc}
-Y^{T} & -W^{T} \\
Z A & Z A_{h}
\end{array}\right] \\
& <0
\end{aligned}
$$

which, by the Schur complement equivalence, gives that for all $h$ satisfying $0<h \leq \bar{h}$

$$
\Lambda(h)<0
$$

This, together with (7), implies

$$
\dot{V}\left(x_{t}\right) \leq-a\|x(t)\|^{2}
$$

where

$$
a=\lambda_{\min }(-\Lambda(h))>0 .
$$

Finally, along a similar line as in the proof of [13, Th. 1], it follows from (8) that the time-delay system $(\Sigma)$ is asymptotically stable for any delay $h$ satisfying $0<h \leq \bar{h}$. This completes the proof.

Remark 1: In the derivation of Theorem 1, two slack variables $Y$ and $W$ are introduced, the purpose of which is to reduce some conservatism in the existing delay-dependent stability conditions. From the proof of Theorem 1 , it can be seen that $\dot{V}_{1}\left(x_{t}\right)$ in (4) remains unaffected when introducing $Y$ and $W$, and hence leads to a more flexible LMI condition in (3). In other words, the rationale behind the introduction of slack variables is to reduce the conservatism of the LMI in Theorem 1 which guarantees the asymptotic stability of a given delay system. The advantages of these introduced variables can be seen from the numerical examples in Section III.

By Theorem 1, we can obtain the following delay-dependent stability result, which has been reported in [9] recently.

$$
\left[\begin{array}{cccc}
P A+A^{T} P+Y+Y^{T}+Q & P A_{h}-Y+W^{T} & -\bar{h} Y & \bar{h} A^{T} Z \\
A_{h}^{T} P-Y^{T}+W & -Q-W-W^{T} & -\bar{h} W & \bar{h} A_{h}^{T} Z \\
-\bar{h} Y^{T} & -\bar{h} W^{T} & -\bar{h} Z & 0 \\
\bar{h} Z A & \bar{h} Z A_{h} & 0 & -\bar{h} Z
\end{array}\right]<0
$$

$$
\begin{aligned}
& \zeta(t, \alpha)=\left[\begin{array}{lll}
x(t)^{T} & x(t-h)^{T} & \dot{x}(\alpha)^{T}
\end{array}\right]^{T} \\
& \Lambda(h)=\left[\begin{array}{ccc}
P A+A^{T} P+Y+Y^{T}+h A^{T} Z A+Q & P A_{h}-Y+W^{T}+h A^{T} Z A_{h} & -h Y \\
A_{h}^{T} P-Y^{T}+W+h A_{h}^{T} Z A & h A_{h}^{T} Z A_{h}-Q-W-W^{T} & -h W \\
-h Y^{T} & -h W^{T} & -h Z
\end{array}\right]
\end{aligned}
$$


TABLE I

COMPARISON OF DELAY-DEPENDENT STABILITY CONDITIONS OF EXAMPLE 1 (FIGURES AS REPORTED IN CORRESPONDING PAPERS)

\begin{tabular}{c|c}
\hline Methods & Maximum $\bar{h}$ allowed \\
\hline Li \& de Souza [5] & 0.8571 \\
\hline Niculescu et al. $[10]$ & 0.9999 \\
\hline Moon et al. $[9]$ & 4.3588 \\
\hline Park [11] & 4.3588 \\
\hline Fridman \& Shaked [1] & 4.47 \\
\hline Theorem 1 in this note & 4.4721 \\
\hline
\end{tabular}

TABLE II

COMPARISON OF ROBUST DELAY-DEPENDENT STABILITY CONDITIONS OF EXAMPLE 2

\begin{tabular}{c|c}
\hline Methods & Maximum $\bar{h}$ allowed \\
\hline Liu \& Su $[7]$ & 0.3025 \\
\hline Li \& de Souza $[6]$ & 0.4428 \\
\hline Lu et al. $[8]$ & 1.3686 \\
\hline Moon et al. $[9]$ & 5.6312 \\
\hline Theorem 2 in this note & $+\infty$
\end{tabular}

Corollary 1: [9] The delay system (1) is asymptotically stable for any delay $h$ satisfying $0<h<\bar{h}$ if there exist matrices $P>0$, $Q>0, X, Y$ and $Z$ such that the following LMI holds:

$$
\left[\begin{array}{ccc}
P A+A^{T} P+\bar{h} X+Y+Y^{T}+Q & P A_{h}-Y & \bar{h} A^{T} Z \\
A_{h}^{T} P-Y^{T} & -Q & \bar{h} A_{h}^{T} Z \\
\bar{h} Z A & \bar{h} Z A_{h} & -\bar{h} Z
\end{array}\right]<0
$$

Proof: By (9), it is easy to see that $Z>0$. Then, it follows from (10) that

$$
X-Y Z^{-1} Y^{T} \geq 0 .
$$

This, together with (9), implies

$$
\begin{aligned}
& {\left[\begin{array}{ccc}
P A+A^{T} P+Y+Y^{T}+Q+\bar{h} Y Z^{-1} Y^{T} & P A_{h}-Y & \bar{h} A^{T} Z \\
A_{h}^{T} P-Y^{T} & -Q & \bar{h} A_{h}^{T} Z \\
- & -\bar{h} Z A
\end{array}\right]} \\
& =\left[\begin{array}{ccc}
P A+A^{T} P+\bar{h} X+Y+Y^{T}+Q & P A_{h}-Y & \bar{h} A^{T} Z \\
A_{h}^{T} P-Y^{T} & -Q & \bar{h} A_{h}^{T} Z \\
\bar{h} Z A & \bar{h} Z A_{h} & -\bar{h} Z
\end{array}\right] \\
& -\left[\begin{array}{ccc}
\bar{h}\left(X-Y Z^{-1} Y^{T}\right) & 0 & 0 \\
0 & 0 & 0 \\
0 & 0 & 0
\end{array}\right]<0
\end{aligned}
$$

which, by the Schur complement formula, gives that

$$
\left[\begin{array}{cccc}
P A+A^{T} P+Y+Y^{T}+Q & P A_{h}-Y & -\bar{h} Y & \bar{h} A^{T} Z \\
A_{h}^{T} P-Y^{T} & -Q & 0 & \bar{h} A_{h}^{T} Z \\
-\bar{h} Y^{T} & 0 & -\bar{h} Z & 0 \\
\bar{h} Z A & \bar{h} Z A_{h} & 0 & -\bar{h} Z
\end{array}\right]<0
$$

Therefore, by Theorem 1, we have that the delay system (1) is asymptotically stable for any delay $h$ satisfying $0<h \leq \bar{h}$.

Remark 2: From the proof of Corollary 1, it is easy to see that Theorem 1 in this note is less conservative than Corollary 1 which was reported in [9, Th. 1]. It is worth pointing out that Theorem 1 is obtained without using the inequality introduced in [9], which was employed to derive upper bounds of some cross terms. Although the inequality in [9] is better than other reported ones when dealing with the delay-dependent stability analysis problem for delay systems in the literature [1], [11], using such a bounding technique still gives relatively conservative results. Without using this bounding technique, Theorem 1 presents a less conservative result.

Remark 3: Compared with the stability condition in [1], it is worth noting that one of the advantages in our note is that the LMI in (3) involves significantly fewer variables than those in [1]. Specifically, in the case when $x(t) \in \mathbb{R}^{n}$, the number of the variables to be determined in (3) is $(n(7 n+3)) / 2$, while in [1] the number of variables is $n(9 n+$ 2 ). That is, the variables in [1] are around 2.6 times more than those in Theorem 1. Therefore, from both mathematical and practical points of view, our condition is more desirable than that in [1] even for some cases when the two methods give the same upper bound on the delay. Another point needs to be mentioned here is that the claim that the result in [1] is less conservative than that in [9, Th. 1] can only be shown by numerical example, while in this note it is theoretically established that Theorem 1 is less conservative than that in [9, Th. 1].

When time-varying norm-bounded parameter uncertainties appear in the time-delay system $(\Sigma)$, that is, system $(\Sigma)$ becomes

$$
\begin{aligned}
(\hat{\Sigma}): \quad \dot{x}(t)= & (A+\Delta A(t)) x(t) \\
& +\left(A_{h}+\Delta A_{h}(t)\right) x(t-h) \\
x(t)= & \phi(t) \quad \forall t \in[-h, 0]
\end{aligned}
$$

where

$$
\left[\Delta A(t) \quad \Delta A_{h}(t)\right]=D F(t)\left[\begin{array}{ll}
E & E_{h}
\end{array}\right]
$$

with $F(t) \in \mathbb{R}^{k \times l}$ being an unknown time-varying matrix function with Lebesgue measurable elements bounded by

$$
F(t)^{T} F(t) \leq I \quad \forall t
$$

we have the following robust delay-dependent stability results.

Theorem 2: The uncertain time-delay system $(\hat{\Sigma})$ is robustly asymptotically stable for any delay $h$ satisfying $0<h \leq \bar{h}$ if there exist a scalar $\epsilon>0$ and matrices $P>0, Q>0, Z>0, Y$, and $W$ such that the LMI shown in (14) at the bottom of the page holds.

The proof of Theorem 2 can be carried out by using Theorem 1 and following a similar line as in [13, Th. 2], and hence it is omitted.

\section{NUMERICAL EXAMPLES}

Example 1: Consider the time-delay system in [5] in the form of (1) with

$$
A=\left[\begin{array}{cc}
-2 & 0 \\
0 & -0.9
\end{array}\right] \quad A_{h}=\left[\begin{array}{cc}
-1 & 0 \\
-1 & -1
\end{array}\right]
$$

The results are compared in Table I. It can be seen that the delay-dependent stability condition in this note is less conservative in the sense

$$
\left[\begin{array}{ccccc}
P A+A^{T} P+Y+Y^{T}+Q+\epsilon E^{T} E & P A_{h}-Y+W^{T}+\epsilon E^{T} E_{h} & -\bar{h} Y & \bar{h} A^{T} Z & P D \\
A_{h}^{T} P-Y^{T}+W+\epsilon E_{h}^{T} E & -Q-W-W^{T}+\epsilon E_{h}^{T} E_{h} & -\bar{h} W & \bar{h} A_{h}^{T} Z & 0 \\
-\bar{h} Y^{T} & -\bar{h} W^{T} & -\bar{h} Z & 0 & 0 \\
\bar{h} Z A & \bar{h} Z A_{h} & 0 & -\bar{h} Z & \bar{h} Z D \\
D^{T} P & 0 & 0 & \bar{h} D^{T} Z & -\epsilon I
\end{array}\right]<0
$$


TABLE III

COMPARISON OF Robust DELAY-DEPENDENT STABILITY CONDITIONS OF EXAMPLE 3

\begin{tabular}{cccccccc}
\hline$\lambda$ & 0.3 & 0.35 & 0.40 & 0.45 & 0.50 & 0.55 & 0.60 \\
\hline $\bar{h}$ by Fridman and Shaked $[2]$ & 0.9288 & 0.8324 & 0.7342 & 0.6242 & 0.4903 & 0.3201 & 0.1027 \\
\hline $\bar{h}$ by Lee et al. $[4]$ & 0.9288 & 0.8324 & 0.7342 & 0.6242 & 0.4903 & 0.3201 & 0.1027 \\
\hline $\bar{h}$ by Theorem 2 in this note & 0.9514 & 0.8711 & 0.7950 & 0.7210 & 0.6426 & 0.5325 & 0.2087 \\
\hline
\end{tabular}

that either the computed maximum delay bound is larger or involving fewer computation variables. In this example, the number of variables used in [1] is 40, and ours is only 17.

Example 2: Consider the uncertain time-delay system in [5]

$$
\begin{array}{ll}
A=\left[\begin{array}{cc}
-2 & 0 \\
1 & -3
\end{array}\right] & A_{h}=\left[\begin{array}{cc}
-1 & 0 \\
-0.8 & -1
\end{array}\right] \\
D=\left[\begin{array}{cc}
0.2 & 0 \\
0 & 0.3
\end{array}\right] & E=E_{h}=\left[\begin{array}{ll}
1 & 0 \\
0 & 1
\end{array}\right] .
\end{array}
$$

For this uncertain system, we compare the results in Table II. It can be shown that this example is robustly stable for any $h>0$ (that is, $\bar{h}$ can be an arbitrarily large positive scalar). The results have demonstrated that the robust delay-dependent stability condition in this note is less conservative.

Example 3: Consider the following uncertain delay system:

$$
\dot{x}(t)=(A+\Delta A(t)) x(t)+\left(A_{h}+\Delta A_{h}(t)\right) x(t-h)
$$

where

$$
A=\left[\begin{array}{cc}
-0.6 & -2.3 \\
0.8 & -1.2
\end{array}\right] \quad A_{h}=\left[\begin{array}{cc}
-0.9 & 0.6 \\
0.2 & 0.1
\end{array}\right]
$$

and the uncertain matrices $\Delta A(t)$ and $\Delta A_{h}(t)$ satisfy

$$
\|\Delta A(t)\| \leq \lambda \quad\left\|\Delta A_{h}(t)\right\| \leq \lambda, \quad \lambda>0 .
$$

This system is of the form in (11)-(13). Then, we can write

$$
D=\lambda I \quad E=E_{h}=I .
$$

Table III gives the comparisons of the maximum allowed delay $\bar{h}$ for various parameter $\lambda$. It can be seen that the results demonstrate that the delay-dependent robust stability condition in this note is less conservative than those in [2] and [4]. Notice that the delay-dependent robust stability results contained in [2] and [4] can be shown to be equivalent to each other.

\section{CONCLUSION}

This note has provided an improved delay-dependent stability condition for linear systems with time-invariant delays in terms of an LMI. The previously used bounding technique has shown to be unnecessary in deriving the delay-dependent stability results. An improved delay-dependent robust stability condition has also been proposed for delay systems with time-varying norm-bounded parameter uncertainties. Numerical examples have demonstrated the effectiveness of the proposed results.

\section{REFERENCES}

[1] E. Fridman and U. Shaked, "A descriptor system approach to $H_{\infty}$ control of linear time-delay systems," IEEE Trans. Autom. Control, vol. 47, no. 2, pp. 253-270, Feb. 2002.

[2] _ "An improved stabilization method for linear time-delay systems," IEEE Trans. Autom. Control, vol. 47, no. 11, pp. 1931-1937, Nov. 2002.

[3] J. K. Hale, Theory of Functional Differential Equations. New York: Springer-Verlag, 1977.

[4] Y. S. Lee, Y. S. Moon, W. H. Kwon, and P. G. Park, "Delay-dependent robust $H_{\infty}$ control for uncertain systems with a state-delay," Automatica, vol. 40, pp. 65-72, 2004.
[5] X. Li and C. E. de Souza, "Criteria for robust stability and stabilization of uncertain linear systems with state-delay," Automatica, vol. 33, pp. 1657-1662, 1997.

[6] — "Delay-dependent robust stability and stabilization of uncertain linear delay systems: A linear matrix inequality approach," IEEE Trans. Autom. Control, vol. 42, no. 8, pp. 1144-1148, Aug. 1997.

[7] P.-L. Liu and T.-J. Su, "Robust stability of interval time-delay systems with delay-dependence," Syst. Control Lett., vol. 33, pp. 231-239, 1998.

[8] C.-Y. Lu, J. S.-H. Tsai, and T.-J. Su, "On improved delay-dependent robust stability criteria for uncertain systems with multiple-state delays," IEEE Trans. Autom. Control, vol. 49, no. 2, pp. 253-256, Feb. 2002.

[9] Y. S. Moon, P. Park, W. H. Kwon, and Y. S. Lee, "Delay-dependent robust stabilization of uncertain state-delayed systems," Int. J. Control, vol. 74, pp. 1447-1455, 2001.

[10] S. I. Niculescu, A. T. Neto, J. M. Dion, and L. Dugard, "Delay-dependent stability of linear systems with delayed state: An LMI approach," in Proc. 34th IEEE Conf. Decision and Control, New Orleans, LA, 1995, pp. 1495-1496.

[11] P. Park, "A delay-dependent stability criterion for systems with uncertain time-invariant delays," IEEE Trans. Autom. Control, vol. 44, no. 4, pp. 876-877, Apr. 1999.

[12] S. Xu, J. Lam, and C. Yang, " $H_{\infty}$ and positive real control for linear neutral delay systems," IEEE Trans. Autom. Control, vol. 46, no. 8, pp. 1321-1326, Aug. 2001.

[13] S. Xu, P. Van Dooren, R. Stefan, and J. Lam, "Robust stability and stabilization for singular systems with state delay and parameter uncertainty," IEEE Trans. Autom. Control, vol. 47, no. 7, pp. 1122-1128, Jul. 2002.

\section{On State-Dependent Dynamic Graphs and Their Controllability Properties}

\author{
Mehran Mesbahi
}

\begin{abstract}
We consider distributed dynamic systems operating over a graph or a network. The geometry of the network is assumed to be a function of the underling system's states-giving it a unique dynamic character. Certain aspects of the resulting abstract structure, having a mixture of combinatorial and system theoretic features, are then studied. In this venue, we will explore an interplay between notions from extremal graph theory and system theory by considering a controllability framework for such state-dependent dynamic graphs.
\end{abstract}

Index Terms-Dynamic graphs, graph controllability, state-dependent graphs, Szemerédi's regularity.

\section{INTRODUCTION}

This note is motivated by system theoretic problems in the area of control and coordination of distributed dynamic systems operating over

Manuscript received February 23, 2004; revised July 19, 2004. Recommended by Associate Editor A. Giua. This material is based upon work supported by the National Science Foundation under Grant 0301753.

The author is with the Department of Aeronautics and Astronautics, University of Washington, Seattle, WA 98195-2400 USA (e-mail: mesbahi@aa.washington.edu).

Digital Object Identifier 10.1109/TAC.2005.843858 